\title{
Sistema de apoio ao diagnóstico da Doença de Chagas baseado em escalogramas e redes neurais auto-organizáveis
}

\author{
João Souza Neto, João Luiz Azevedo de Carvalho, Adson Ferreira da Rocha, \\ Luiz Fernando Junqueira Junior, Francisco Assis de Oliveira Nascimento*
}

Resumo Introdução: A Doença de Chagas é uma endemia rural, prevalente em grande parte da América Central e América do Sul e, aproximadamente, metade dos pacientes contaminados com o parasita Trypanosoma cruzi não apresentam sinais clínicos, eletrocardiográficos e radiológicos de envolvimento cardíaco. Este trabalho, entretanto, propõe uma técnica de auxílio ao diagnóstico da Doença de Chagas baseada em sinais de eletrocardiografia, que extrai informações relevantes desses sinais. Métodos: Duas abordagens são estudadas e implementadas. Ambas utilizam sinais de variabilidade da frequência cardíaca (VFC) e classificação por meio de rede neural, mais especificamente, o mapa auto-organizável de Kohonen. A VFC, que reflete a modulação neural autonômica simpática e parassimpática do coração, é avaliada com base em séries contínuas de intervalos RR do ECG convencional registradas durante 5 minutos. Na primeira abordagem, indicadores estatístico-temporais obtidos diretamente dos sinais de VFC são utilizados como entrada da rede neural para treinamento e teste do método de classificação. Na segunda proposta, são utilizados escalogramas wavelet com função de base DoG (derivative of Gaussian) para avaliação dos sinais de VFC. Indicadores obtidos dos escalogramas são utilizados como entrada da rede neural no treinamento e no teste do algoritmo. Os mapas topológicos de Kohonen são utilizados para comparar a capacidade dos indicadores calculados dos sinais de VFC em discriminar pacientes chagásicos cardiopatas, chagásicos indeterminados e indivíduos normais. Os indicadores temporais convencionais e os indicadores escalográficos são comparados. Resultados: Os resultados mostram que os indicadores escalográficos têm poder discriminatório estatisticamente superior aos indicadores temporais convencionais. Em particular, a potência média da densidade de potência do escalograma na banda de altas frequências mostrou ser estatisticamente o indicador de maior poder discriminatório ( $p<0,05$ para os 3 casos). Conclusão: A metodologia proposta mostrou-se capaz de distinguir entre indivíduos normais, chagásicos cardiopatas e chagásicos indeterminados. Os índices escalográficos propostos mostraram maior capacidade classificatória que os índices temporais tradicionais.

Palavras-chave Doença de Chagas, Escalograma, Wavelets, Redes neurais.

\section{Computer-aided system for diagnosis of Chagas' disease based on scalograms and self-organizing neural networks}

Abstract Introduction: Chagas' disease is an endemic rural disease prevalent in much of Central America and South America, and approximately half of the patients infected with the parasite Trypanosoma cruzi show no clinical, electrocardiographic and radiological cardiac involvement. This paper, however, proposes a technique for the diagnosis of Chagas'disease based on ECG signals, which extracts relevant information from these signals. Methods: Two approaches are studied and implemented. Both approaches use heart rate variability (HRV) signals, and classification by a neural network, more specifically, the Kohonen selforganizing map. The HRV, which reflects sympathetic and parasympathetic autonomic neural modulation of the heart, is evaluated based on continuous series of RR intervals, calculated from 5-minute records of conventional ECG. In the first approach, statistical/temporal indexes obtained directly from the HRV signals are used as neural network inputs for training and testing of the classification method. In the second approach, derivative of Gaussian (DoG) wavelet scalograms are used to evaluate the HRV signals. Scalographic indexes are used as neural network inputs for training and testing of the algorithm. Kohonen topological maps are used to compare the ability of these HRV indicators of discriminating between patients with Chagas heart disease, Chagas indeterminate heart disease, and normal subjects. Conventional temporal indicators and indicators obtained from DoG scalograms are compared. Results: Results of the application of the proposed methods to HRV signal databases, and performance comparisons, are presented. The results show that scalographic indicators have superior discriminatory power than conventional time-domain indicators. Based on an analysis of statistical significance, we show that the average power of the high-frequency band of the scalogram power spectral density is the indicator with greatest discriminatory power $(p<0,05$ for all 3 cases). Conclusion: The proposed method has the ability to discriminate between normal subjects, subjects with Chagas cardiomyopathy, and subjects with the indeterminate form of Chagas' disease. It was observed that scalographic neural networks present greater discrimination ability than temporal neural networks.

Keywords Chagas'disease, Scalogram, Wavelets, Neural networks. 


\section{Introdução}

A doença de Chagas é uma endemia rural, prevalente em grande parte da América Central e América do Sul. É também encontrada no Caribe, sul dos Estados Unidos e em alguns países da Europa. No Brasil, apesar da queda drástica do número de novos casos anuais, não se pode apontar a erradicação da doença. Aproximadamente, metade dos pacientes contaminados com o parasita Trypanosoma cruzi não apresentam sinais clínicos, eletrocardiográficos e radiológicos de envolvimento cardíaco. Cerca de 30\% apresenta alguma manifestação de comprometimento do coração, com ou sem sintomas clínicos, detectada em bases clínicas e por meio de testes diagnósticos diversos (Junqueira Jr, 2012).

O diagnóstico da doença de Chagas, especialmente em regiões remotas, pode ser facilitado por tecnologias de informática médica. Estas proporcionam, ao profissional de medicina, facilidades computacionais dedicadas, que auxiliam o atendimento de pacientes, como o acesso a banco de dados de imagens médicas e a prontuários eletrônicos, suporte a emissão de laudos especializados e suporte a colegas situados em regiões remotas do país, muitas vezes, em localidades sem as devidas condições mínimas necessárias ao atendimento da saúde humana. Tecnologias voltadas ao apoio da decisão médica constituem uma linha de pesquisa e de desenvolvimento de interesse das políticas do Governo Brasileiro no segmento da saúde.

Métodos computacionais de auxílio complementar ao diagnóstico e classificação mais apurados de pacientes acometidos pela doença de Chagas, com o objetivo de avaliar danos ao sistema cardiovascular, foram objeto de estudo nas últimas duas décadas, a exemplo da análise da variabilidade da frequência cardíaca (Correia et al., 2007; Vasconcelos e Junqueira Jr, 2009, 2011). A aplicação de redes neurais e lógica fuzzy em cardiologia é ampla e variada, incluindo desde a localização de infartos do miocárdio a partir do desnivelamento do segmento ST nas 12 derivações do ECG, por meio de quantização vetorial com aprendizagem (Reinhardt et al., 1996), e o diagnóstico de infartos do miocárdio com redes neurais do tipo percéptron multi-camadas (multilayer perceptron, ou MLP) com algoritmo de treinamento por backpropagation (Cházaro et al., 1998), até a análise de arritmias ventriculares em sinais de ECG, utilizando redes neurais do tipo funções de base radiais (radial basis functions) e o algoritmo fuzzy c-means (Heidari et al., 1998).

Bravo et al. (1997), por exemplo, analisaram sinais eletrocardiográficos (ECG) de alta resolução, anormais, coletados de pacientes chagásicos, usando a transformada discreta de Hartley. Nesse trabalho, foi proposta uma técnica para detecção de pacientes com enfermidade miocárdica generalizada, com base na distribuição de energia dos potenciais tardios, avaliada no domínio transformado. Guillén et al. (2000), por sua vez, mostraram que parâmetros de variabilidade da frequência cardíaca (VFC) obtidos por meio de modelos dinâmicos não-lineares e caóticos podem ser usados para discriminar indivíduos chagásicos de indivíduos normais.

Propostas de técnicas não invasivas, com o uso de inteligência artificial para classificar pacientes chagásicos em diferentes estágios da doença, também são observadas na literatura (Marmol-Herrera e Warwick, 2001). Nesse trabalho, indicadores extraídos da forma de onda de sinais de ECG são utilizados como entrada de redes neurais de Kohonen. O trabalho enfatiza o dimensionamento adequado das redes neurais de forma a obter melhor classificação. Nos resultados obtidos, aparecem "zonas de incertezas", as quais reduzem a acurácia do processo.

Abordagem integrando redes neurais e transformadas wavelet também já foi investigada (Carranza e Andina, 2002). Nesse trabalho, primeiramente, o sinal de ECG é processado por meio da transformada de Haar, que procura evidenciar transitórios rápidos associados a arritmias típicas da doença de Chagas. Uma rede neural MLP é aplicada para estimar a probabilidade de infecção do paciente, que é apresentada ao profissional especialista no diagnóstico. Limitações da transformada de Haar com respeito à representação no domínio transformado (capacidade de descorrelação de sinais) não são avaliadas.

Pueyo et al. (2007) estudaram a inclinação dos complexos QRS para determinar o grau de dano miocárdico em pacientes chagásicos crônicos. Seus resultados indicam que pacientes chagásicos apresentam inclinação do complexo QRS significantemente menor que em sujeito saudáveis. Outra técnica baseada na caracterização de pacientes chagásicos por códigos corretores de erros foi proposta por Escalera et al. (2008). Nesse trabalho, foi utilizado o mesmo banco de dados construído por Pueyo et al. (2007), mas são apresentados resultados de acurácia ligeiramente superior. Por sua vez, Correa et al. (2010) propuseram a análise da eletrocardiografia vetorial para a caracterização de diferenças de alterações cardíacas em pacientes chagásicos crônicos com diferentes graus de dano miocárdico, a partir de avaliações estatísticas de variações do complexo QRS.

Vizcardo et al. (2007) descreveram uma técnica que mostra alterações espectrais nos índices de VFC associadas a doença de Chagas. Nesse trabalho, 
foram estudados indivíduos saudáveis e indivíduos com e sem alterações no ECG que apresentavam diagnóstico positivo de doença de Chagas. $\mathrm{O}$ trabalho propôs uma representação e uma base ortogonal de forma a caracterizar esses três perfis. A acurácia dos resultados apresentados em função da sensibilidade dos parâmetros discriminatórios (RMSSD e pNN50) indicaram probabilidade maior que $60 \%$ em discriminar o indivíduo em relação aos três grupos estabelecidos.

A maioria das investigações baseadas nas características espectrais dos sinais de VFC ainda tem sido realizada com a transformada discreta de Fourier ou com o modelo auto-regressivo. Entretanto, a transformada wavelet mostra-se mais adequada que estas técnicas para a representação de sinais não estacionários. Esta representa os sinais com diferentes resoluções espectrais e temporais, permitindo a identificação de fragmentos com grandes e pequenas escalas (baixas e altas frequências, respectivamente). No caso específico dos sinais de VFC, a razão entre as frequências características de interesse sugere o uso das wavelets como ferramenta de análise espectral. Do extremo inferior da banda VLF (very low frequency, ou frequência muito baixa) - $0,003 \mathrm{~Hz}$ - ao extremo superior da banda HF (high frequency, ou alta frequência) - $0,4 \mathrm{~Hz}$ - desses sinais, encontram-se componentes com razões de frequência com valores superiores a 100:1. Essa característica sugere o uso de ferramentas de estimação espectral com resolução frequencial variável (logarítmica, por exemplo) para compactar a faixa dinâmica da banda de frequências (Lotric et al., 2000). Isso é possível com uso da transformada wavelet. As distribuições tempo-escala obtidas por meio da transformada wavelet são chamadas de espectrogramas wavelet ou escalogramas (Souza Neto, 2003).

O presente trabalho apresenta uma técnica de apoio ao diagnóstico clínico para doença de Chagas subdividida em duas etapas: (i) extração da assinatura espectral de interesse, a partir do escalograma wavelet calculado do sinal de VFC; e (ii) classificação por meio de rede neural, mais especificamente, o mapa auto-organizável de Kohonen. Duas abordagens são avaliadas. Na primeira abordagem, índices estatísticotemporais obtidos diretamente dos sinais de VFC são utilizados como entrada da rede neural para treinamento e teste do método de classificação. $\mathrm{Na}$ segunda proposta, são utilizados escalogramas wavelet com função de base DoG (derivative of Gaussian) para avaliação dos sinais de VFC. Índices obtidos dos escalogramas são utilizados como entrada da rede neural no treinamento e no teste do algoritmo. Os mapas topológicos de Kohonen (1995) são utilizados para comparar a capacidade dos indicadores calculados dos sinais de VFC de discriminar pacientes chagásicos cardiopatas, chagásicos indeterminados e indivíduos normais. Os indicadores temporais convencionais e os indicadores escalográficos são comparados. Resultados de aplicação dos métodos em banco de sinais e comparação de desempenho também são apresentados.

\section{Métodos}

Este trabalho compara duas abordagens para auxílio ao diagnóstico de pacientes com enfermidade de Chagas. No contexto da proposta, existem três possibilidades para classificação: indivíduos normais; chagásicos cardiopatas; ou chagásicos indeterminados. $\mathrm{Na}$ abordagem de classificação dos padrões, utiliza-se o mapa auto-organizável de Kohonen com dois tipos de índices como entradas da rede neural: (i) aqueles provenientes de medições estatísitico-temporais tradicionais do sinal de VFC; e (ii) os provenientes de escalogramas DoG. Avaliações de desempenho e comparação dos resultados entre esses dois algoritmos de classificação são apresentados.

Na Figura 1, são apresentados os diagramas de blocos simplificados dos algoritmos propostos neste trabalho. Em ambos os algoritmos, primeiramente tem-se a construção do sinal de VFC (Figura 1a), que se resume à detecção das ondas $\mathrm{R}$, cálculo dos intervalos RR e remoção e correção de intervalos associados a batimentos ectópicos. No primeiro algoritmo (Figura 1b), são extraídas informações (índices) estatísticas e temporais do sinal de VFC, as quais são utilizadas como entrada para o módulo de classificação por meio do mapa auto-organizável de Kohonen. No segundo algoritmo (Figura 1c), o sinal de VFC passa por um pré-processamento, realizado em duas etapas: (i) uma interpolação por splines cúbicas com amostragem a uma taxa de $4 \mathrm{~Hz}$, com o objetivo de uniformizar o espaçamento temporal entre as amostras do sinal; e (ii) remoção (subtração) do valor médio do sinal, o qual poderia mascarar as demais componentes do sinal. O sinal pré-processado é utilizado como entrada para a análise de distribuições tempo-escala por meio da transformada wavelet (escalograma), que minimiza a redundância intrínseca do sinal de VFC, compactando a energia da assinatura espectral de interesse. Os índices extraídos do escalograma são utilizados como entradas para o módulo de classificação.

\section{Indivíduos e sinais estudados}

Foram utilizadas séries de intervalos RR com comprimento de sinal de aproximadamente 300 segundos (5 minutos), obtidas da base de dados 
(a)

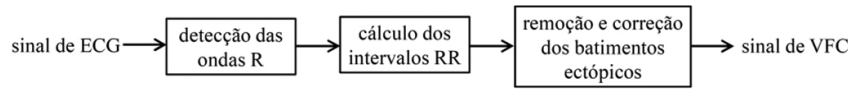

(b)

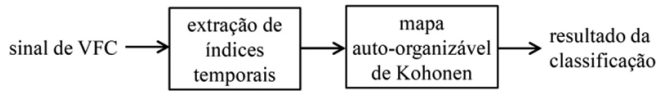

(c)

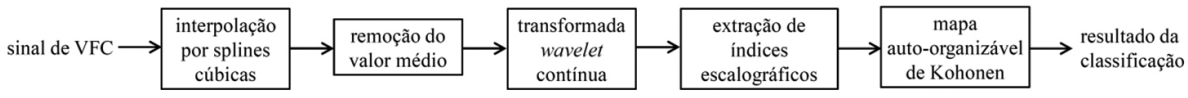

Figura 1. Diagramas de blocos do: (a) processo de construção do sinal de VFC; (b) processo de classificação com índices temporais; e (c) processo de classificação com índices escalográficos.

Figure 1. Block diagrams for: (a) HRV signal construction process; (b) classification process using temporal indexes; and (c) classification process using scalographic indexes.

do Laboratório Cardiovascular da Faculdade de Medicina da Universidade de Brasília. Foram estudados três grupos de pacientes, em posição supina: o grupo controle, com 28 indivíduos normais; o grupo de chagásicos cardiopatas, com 13 indivíduos; e o grupo de chagásicos indeterminados, com 11 indivíduos com a forma indeterminada da doença de Chagas. Todos os procedimentos foram aprovados pelo comitê de ética da Faculdade de Ciências da Saúde da Universidade de Brasília.

\section{Sinal de variabilidade da frequência cardíaca}

A análise do sinal de VFC no domínio escala-frequência permite a identificação e a avaliação quantitativa da atividade da função autonômica cardíaca. No espectro de potência do sinal de VFC, encontramos: componentes na faixa de $0,15 \mathrm{~Hz}$ a $0,40 \mathrm{~Hz}$ - denominada banda HF (high frequency, ou alta frequência), que reflete a modulação cardíaca parassimpática (vagal) associada à arritmia sinusal respiratória; componentes na faixa de $0,04 \mathrm{~Hz}$ a $0,15 \mathrm{~Hz}$ - denominada banda LF (low frequency, ou baixa frequência), relacionadas às modulações autonômicas simpática e parassimpática simultaneamente, associadas ao controle barorreflexo da pressão arterial; e componentes na faixa de 0,003 $\mathrm{Hz}$ a $0,04 \mathrm{~Hz}$ - denominada banda VLF (very low frequency, ou frequência ultra baiza), associadas a mecanismos regulatórios lentos, como termorregulação, vasomotricidade, atividade do sistema reninaangiotensina-aldosterona e outros não estacionários (Wiklund e Akay, 1998; Kleiger et al., 2005).

A variabilidade da frequência cardíaca na região espectral LF pode ser dividida em fases periódicas e não periódicas. As variações não periódicas estão, geralmente, relacionadas a atividades fisiológicas e a situações de grande carga emocional. As variações periódicas, por sua vez, têm sido observadas em pacientes com distúrbios cardiopulmonares e têm sido consideradas sinais de perda da atividade homeostática
(Sapoznikov et al., 1994). A variabilidade da frequência cardíaca na região LF contribui com mais de $90 \%$ da potência total do sinal de VFC.

A componente VLF, por sua vez, também é chamada de componente $1 /$ f por causa do aumento da sua energia espectral com a diminuição da frequência. A componente $1 / \mathrm{f}$ tem natureza fractal, isto é, a sua potência espectral obedece a uma lei de potência inversa fractal (Yang e Liao, 1997).

Algumas alterações dos ritmos do sinal de VFC têm sido associadas a condições patológicas (Kleiger et al., 2005). Em particular, o aumento da energia da componente LF tem sido associado a ataques isquêmicos e outras patologias (Bifulco et al., 2000). A avaliação da função autonômica parassimpática a partir da análise espectral é obtida pela medição da área sob a curva do espectro de potência do sinal de VFC na banda HF. A atividade simpática é mais difícil de quantificar utilizando essa metodologia. Um conceito melhor é o do equilíbrio simpato-vagal, que leva em consideração as influências simpáticas e parassimpáticas recíprocas e não recíprocas sobre a frequência cardíaca, com a medição da razão das energias LF e HF (razão LF/HF). As razões das potências espectrais do sinal de VFC definidas por Bootsma et al. (1994) - LF/(LF+HF), $\mathrm{LF} /(\mathrm{VLF}+\mathrm{LF}+\mathrm{HF})$ e $(\mathrm{VLF}+\mathrm{LF}) /(\mathrm{VLF}+\mathrm{LF}+\mathrm{HF})-$ permitem a análise comparada das potências das diversas componentes do espectro do sinal de VFC.

\section{O escalograma}

O espectrograma wavelet, ou escalograma, é definido como o quadrado da magnitude da transformada wavelet contínua (continuous wavelet transform, ou CWT). Este representa a distribuição de energia do sinal no plano tempo-escala, expressa em potência por unidade de frequência $\left(\mathrm{ms}^{2} / \mathrm{Hz}\right)$, de maneira análoga ao espectrograma de Fourier. 
A CWT de uma função contínua $f(t)$ quadraticamente integrável em uma escala $\tau \in \mathfrak{R}^{+} \mathrm{e}$ com um deslocamento $\tau \in \mathfrak{R}$ é:

$$
F_{\psi}(\tau, s)=\frac{1}{\sqrt{s}} \int_{-\infty}^{\infty} f(t) \psi *\left(\frac{t-\tau}{s}\right) d t
$$

onde $\psi(t)$ é chamada de wavelet mãe $\mathrm{e}^{*}$ denota conjugação complexa. Então, para um sinal discreto no tempo $f[n]$, com intervalo de amostragem $T_{s}$, a CWT é definida como:

$$
F_{\psi}(\tau, s)=\frac{1}{\sqrt{s}} \sum_{n} f[n] \int_{n T_{s}-T_{s} / 2}^{n T_{s}+T_{s} / 2} \psi^{*}\left(\frac{t-\tau}{s}\right) d t
$$

Neste trabalho, a CWT foi calculada usando translações de uma em uma amostra e para $s=s_{0} 2^{\mathrm{k}}$, onde $s_{0}=2 T_{s}$ e com $k$ variando de 0 a 7 , em passos de $1 / 16$. Em outras palavras, a menor escala corresponde a uma função wavelet de apenas duas amostras ( 0,5 segundos), enquanto que a maior escala corresponde a uma função wavelet com 256 amostras (64 segundos); e o tamanho da escala é variado de modo exponencial.

O escalograma pode ser definido como uma versão affine suavizada da distribuição de WignerVille (Rioul e Flandrin, 1992). Souza Neto (2003) analisou o nível de ocupação espectral da distribuição de Wigner-Ville, do espectrograma de Fourier, do escalograma de Morlet e do escalograma DoG nas bandas VLF, LF e HF do espectro do sinal de VFC. Este é um critério importante, dadas as elevadas razões entre as frequências características de interesse do sinal de VFC. A wavelet com função de base DoG apresentou uma resolução superior às outras distribuições para esta aplicação específica, devido à elevada granularidade da sua estrutura tempo-escala (Souza Neto, 2003). Isto ocorre porque a DoG é uma função real e retorna apenas um único componente frequencial, sendo mais adequada para isolar picos e descontinuidades. Além disso, a DoG captura as oscilações positivas e negativas da série temporal como picos separados do espectro de potência. A wavelet DoG mãe é definida como:

$$
\psi(t)=\frac{(-1)^{m+1}}{\sqrt{\Gamma\left(m+\frac{1}{2}\right)}} \frac{d^{m}}{d t^{m}} e^{-t / 2}
$$

onde $m$ é a ordem da derivada da função Gaussiana e $\Gamma(z)$ é a função gama. Neste trabalho, foi usado $m=10$.

\section{Análise escalográfica da função autonômica cardíaca do chagásico}

Na doença de Chagas, o parasita afeta com intensidade variável a musculatura atrial e ventricular, o tecido de excitação-condução elétrica e a inervação autonômica intrínseca, particularmente a inervação parassimpática (Junqueira Jr, 2012). Estudos baseados em necropsias mostraram, em chagásicos cardiopatas, grau de denervação parassimpática entre $52 \%$ e $94 \%$ e simpática em torno de 37\% (Esteller et al., 1994). Portanto, espera-se observar, nos escalogramas, principalmente depressão da função parassimpática característica da doença de Chagas em suas diferentes formas clínicas, o que pode determinar ou contribuir para a ocorrência de arritmias, morte súbita e progressão da disfunção contrátil (Vasconcelos e Junqueira Jr, 2009; Junqueira Jr, 2012).

Os escalogramas com função de base DoG apresentam uma distribuição tempo-escala com rico conteúdo espectral. As Figuras 2 e 3 apresentam três exemplos de escalograma DoG, calculados para trechos de sinais de VFC de um indivíduo normal (Figura 2a), de um paciente chagásico cardiopata (Figura 3a) e de um paciente chagásico indeterminado (Figura 3b), na posição supino. No escalograma do indivíduo normal (Figura 2b), a componente LF do sinal é mapeada com clareza. No escalograma do indivíduo chagásico cardiopata (Figura 3c), o conteúdo frequencial das bandas VLF e HF é mapeado com clareza, mas nota-se também a ausência da componente LF (função simpática). Já no escalograma do indivíduo chagásico indeterminado (Figura 3d), além da ausência da componente LF, pode-se notar que a distribuição de energia em altas frequências é mais esparsa e fragmentada, demonstrando a perda da capacidade de modulação da função autonômica cardíaca na faixa de altas frequências (banda HF), relacionada ao sistema parassimpático.

A densidade espectral de potência $F(s)$ é obtida a partir da transformada wavelet DoG - calculada substituindo a Equação 3 na Equação 2 - do sinal de HRV pré-processado, como:

$$
F(s)=\frac{1}{T} \int_{0}^{T}\left|F_{\psi}(\tau, s)\right|^{2} d \tau,
$$

onde $T$ é o tamanho da janela de observação, isto é, o período total de aquisição (Souza Neto, 2003).

Três exemplos de espectros de potência wavelet são apresentados nas Figuras 2 e 3. Para o indivíduo normal (Figura 2c), nota-se que a potência é bem distribuída nas bandas VLF, LF e HF do espectro, com um pico na componente LF. Para o indivíduo chagásico cardiopata (Figura 3e), nota-se que o espectro é plano, tanto na banda VLF quanto na banda LF. Já para o indivíduo chagásico indeterminado (Figura 3f), observa-se, que o espectro apresenta componente LF substancialmente atenuada, forte depressão da curva na banda HF e uma distribuição desigual de energia em todo o espectro. A baixa amplitude na banda HF sugere a perda de capacidade de modulação "fina" 
(a)
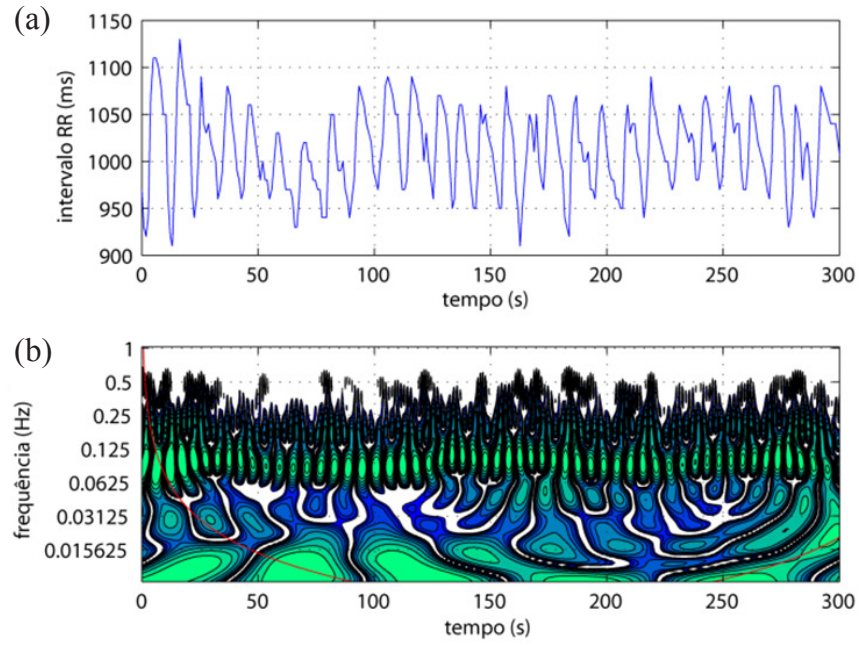

(c)

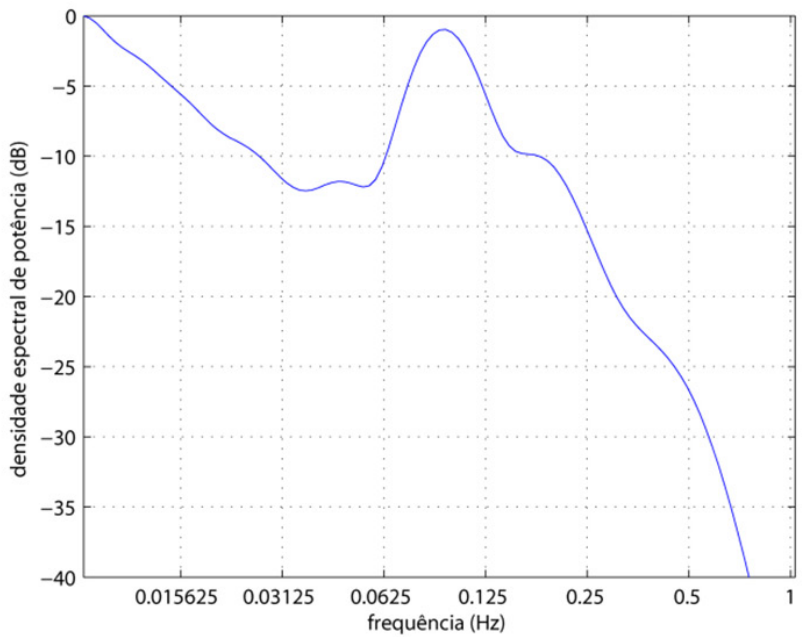

Figura 2. Análise escalográfica de um segmento de cinco minutos de um sinais de VFC de um indivíduo normal: (a) série de intervalos RR; (b) escalograma (com amplitudes em escala logarítmica); e (c) densidade espectral de potência, obtida do escalograma.

Figure 2. Scalographic analysis of a five-minute segment of an HRV signal from a normal subject: (a) RR interval time series; (b) scalogram (amplitudes are in log scale); and (c) power spectral density, calculated from the scalogram.

da função autonômica cardíaca, característica de depressão da modulação vagal.

O processo de classificação utilizando informações escalográficas é realizado computando-se a potência média e a faixa dinâmica de potência nas bandas VLF, LF e HF. Esses índices são utilizados como entradas da rede neural.

\section{O mapa auto-organizável de Kohonen}

O mapa auto-organizável de Kohonen (MAK) pertence à classe das redes neurais sem treinamento supervisionado e foi desenvolvido tendo como modelo o processo biológico de reconhecimento de padrões visuais (Kohonen, 1995). O MAK mapeia relações estatísticas complexas e não-lineares entre dados multidimensionais em relações geométricas simples, em uma grade regular de representação de baixas dimensões. Como ocorre uma compressão da informação nesse processo - preservando-se, entretanto, as mais importantes relações topológicas e métricas entre os dados originais na grade de representação, é possível produzir representações abstratas dos itens de dados (Kohonen, 1995). Esses dois aspectos (visualização e abstração) podem ser utilizados de várias maneiras em aplicações complexas, tais como diagramas de estado de processos e máquinas, gráficos de similaridade de tabelas estatísticas de aplicações de data mining, etc.

O algoritmo MAK computa os modelos de forma que estes representem de maneira ótima o domínio das observações (vetores de entrada). Os modelos 
(a)

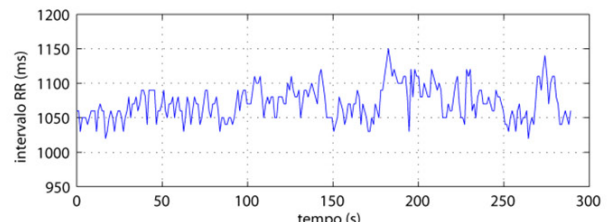

(c)

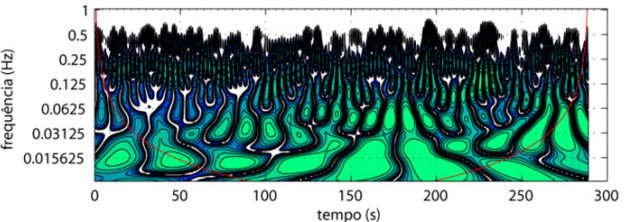

(e)

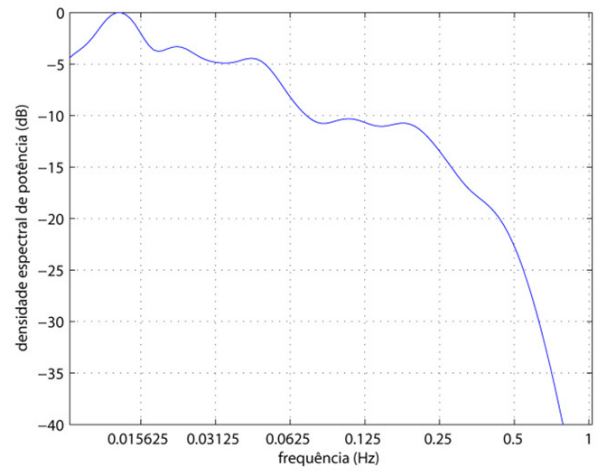

(b)

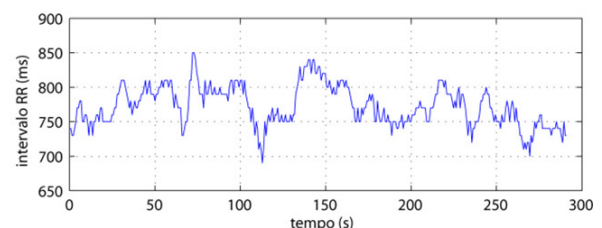

(d)

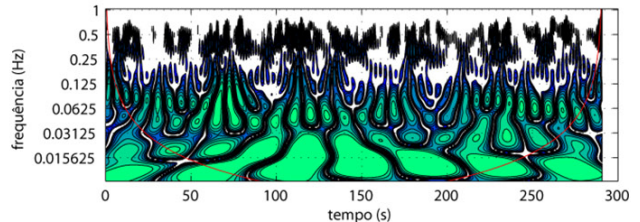

(f)

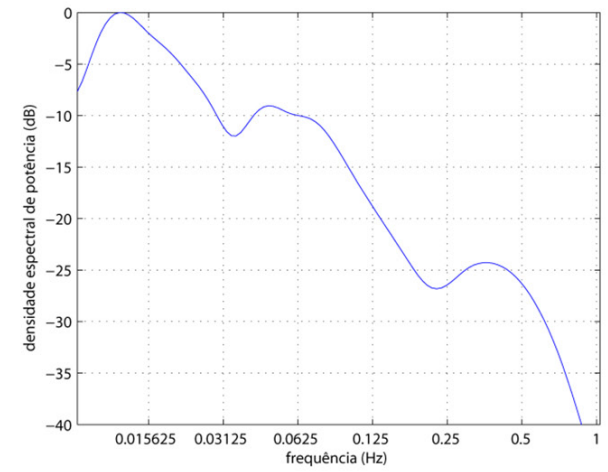

Figura 3. Análise escalográfica de segmentos de cinco minutos de sinais de VFC de indivíduos chagásicos cardiopata (coluna da esquerda) e indeterminado (coluna da direita): (a,b) séries de intervalos RR; $(c, d)$ respectivos escalogramas (com amplitudes em escala logarítmica); e $(\mathrm{e}, \mathrm{f})$ densidades espectrais de potência, obtidas dos escalogramas.

Figure 3. Scalographic analysis of five-minute segments of HRV signals from patients with Chagas cardiomyopathy (left column) and with the indeterminate form of Chagas' disease (right column): (a,b) RR interval time series; $(c, d)$ corresponding scalograms (amplitudes are in log scale); and (e,f) power spectral densities, calculated from the scalograms.

são organizados em uma ordem bidimensional, na qual modelos semelhantes estão mais próximos uns dos outros do que de modelos dissimilares. Nesse aspecto, o MAK é um gráfico de similaridades e, também, um diagrama de agrupamentos.

O algoritmo MAK é calculado num processo de regressão recursivo e não paramétrico. A regressão de um conjunto ordenado de vetores de modelo, $m_{i} \Re^{n}$, no espaço dos vetores de entrada, $x \in \mathfrak{R}^{n}$, é realizada pelo processo:

$m_{i}(t+1)=m_{i}(t)+h_{c(x), i}\left(x(t)-m_{i}(t)\right)$,

onde $t$ é o índice de amostras dos passos da regressão. A regressão é realizada recursivamente para cada apresentação de uma amostra de $x$. O índice $c$ (do nó vencedor) é definido pela condição:

$$
\left\|x(t)-m_{c}(t)\right\| \leq\left\|x(t)-m_{i}(t)\right\| \forall i
$$

A função de vizinhança, $h_{c(x), i}$, opera como um kernel de suavização variante no tempo e a sua posição depende das condições da Equação 5. Essa função decresce com a distância entre o $i$-ésimo e o $c$-ésimo modelos no mapa da grade. A função de vizinhança é, geralmente, Gaussiana:

$h_{c(x), i}=\alpha(t) e^{-\frac{\left\|r_{i}-r_{c}\right\|^{2}}{2 \sigma^{2}(t)}}$

onde $\alpha(t) \in[0,1]$ é o fator de aprendizado, que decresce monotonicamente com os passos de regressão; $r_{i} \in \mathfrak{R}^{2} \mathrm{e} r_{c} \in \mathfrak{R}^{2}$ são as posições vetoriais na grade de representação; e $\sigma(t)$ corresponde à largura da função de vizinhança, que também decresce monotonicamente com os passos de regressão.

$\mathrm{Na}$ aplicação de classificação de pacientes em normais, chagásicos cardiopatas e chagásicos indeterminados, a partir dos índices escalográficos e dos índices temporais, o MAK foi utilizado como mapa de agrupamentos, definindo regiões do mapa como representativas de uma das categorias selecionadas.

\section{Metodologia experimental}

A classificação neural foi realizada com duas abordagens distintas:

- Técnica 1: Índices temporais são utilizados como entradas da rede neural. Os índices 
temporais obtidos das séries de intervalos RR foram: média, desvio padrão, coeficiente de variação, pNN50 e RMSSD. O coeficiente de variação é a razão entre o desvio padrão e a média; o pNN50 é a porcentagem das diferenças absolutas entre intervalos RR normais sucessivos que excedem $50 \mathrm{~ms}$; e o RMSSD é o valor eficaz das diferenças entre intervalos RR sucessivos. Essa abordagem, de se procurar a indicação de diagnóstico dos pacientes chagásicos por meio de índices diretamente computados a partir do sinal de VFC, se aproxima mais das propostas encontradas na literatura (Correa et al., 2010; Marmol-Herrera e Warwick, 2001; Vizcardo et al., 2007).

- Técnica 2: Índices escalográficos são utilizados como entradas da rede neural. Para o escalograma, definiu-se a densidade de energia como o indicador representativo de quão densa é a distribuição de energia no espectro do sinal. Quanto maior for a ocupação espectral pelo sinal, maior é esse índice. $\mathrm{O}$ valor limite para essa medição, escolhido heuristicamente, foi de $-2,3 \mathrm{~dB}$ de amplitude de raia. Já para a densidade espectral de potência, os indicadores escolhidos foram a potência média e a faixa dinâmica de potência nas bandas VLF, LF e HF. A faixa dinâmica de potência consiste na diferença entre o valor de pico e o valor de vale de potência na banda em análise. Com esses índices, pretende-se avaliar quão plana é a distribuição de energia nas bandas e de que forma a energia total do sinal de VFC é distribuída entre elas.

Para o mapa auto-organizável de Kohonen, foram definidos mapas hexagonais com dimensões $16 \times 32$. O treinamento decorreu durante 50 épocas (cada época sendo uma passada completa no conjunto de vetores de entrada). Utilizou-se vizinhança com formato Gaussiano e a taxa de treinamento inicial foi de 0,5. Do universo de vetores, foram utilizados $75 \%$ para treinamento e $25 \%$ para teste.

\section{Resultados}

As Figuras 4a e 4b mostram os mapas obtidos após o treinamento das redes neurais temporal e escalográfica, respectivamente. Observa-se, para a rede neural temporal, que os vetores dos pacientes chagásicos indeterminados formaram três grupos distintos, o que demonstra uma capacidade de agrupamento pobre. No caso da rede neural escalográfica, cada tipo de vetor formou um grupo único, o que demonstra a capacidade discriminatória superior dos indicadores escalográficos. A Figura 4 mostra ainda a grade do mapa (Figuras 4c e 4d), a matriz de distâncias entre os vetores de entrada e os vetores protótipos (modelos) (Figuras $4 \mathrm{e}$ e 4f), os vetores protótipos (Figuras $4 \mathrm{~g}$ e $4 \mathrm{~h}$ ), e os dados obtidos após o treinamento superpostos aos vetores protótipos (Figuras 4i e 4j). Na Figura 4i, observa-se que os vetores protótipos (modelos) e os vetores de entrada encontram-se espalhados, o que demonstra que a rede neural temporal não conseguiu modelar a contento os dados apresentados. Já para a rede escalográfica (Figura 4j), o gráfico mostra-se mais denso, o que indica um melhor mapeamento das observações.

A Tabela 1 mostra os resultados obtidos para as redes neurais escalográficas e temporais. O indicador de qualidade best matching unit (BMU) (Kiviluoto, 1996; Kohonen, 1995) retrata a diferença entre os modelos produzidos pela rede e o conjunto de vetores de entrada, isto é, quão fiel é a representação da grade neural. O algoritmo para cálculo do indicador simplesmente computa a distância Euclidiana entre cada vetor de entrada e o vetor que melhor o representa no mapa. Quanto maior o valor do indicador BMU, melhor foi a representação neural. Já o erro de classificação demonstra, após um treinamento com 50 épocas, quão bem a rede neural classificou os padrões de entrada. Assim, os resultados apresentados mostram que as redes neurais escalográficas apresentaram resultados superiores às redes neurais temporais, o que comprova que, para o universo analisado, os índices escalográficos foram melhores que os índices temporais para a classificação de pacientes em normais, chagásicos cardiopatas e chagásicos indeterminados, a partir de índices relacionados ao seus sinais de VFC.

Além da análise neural, para cada série de índices, avaliou-se a normalidade da série e calculou-se o $p$, que compara a mediana de duas populações com relação à variabilidade das distribuições. Foi utilizado, aqui, o teste não paramétrico de Mann-Whitney, devido ao fato de ter-se poucas amostras, com medianas que

Tabela 1. Comparação entre as redes neurais temporal e escalográfica.

Table 1. Comparison between temporal and scalographic neural networks.

\begin{tabular}{lcc}
\hline & Indicador de qualidade BMU & Erro de classificação \\
\hline Rede neural temporal & 0,469 & 4,284 \\
Rede neural escalográfica & 0,756 & 2,439 \\
\hline
\end{tabular}


não coincidem com as médias. Definiu-se um valor de significância de 0,05 , o que implica que valores de $p$ menores que esse limite definem diferenças estatisticamente significativas, isto é, que não são fruto do acaso. Nessa análise, cujos resultados são apresentados na Tabela 2, observou-se que o índice com maior poder discriminatório é a potência média na banda HF, que é capaz de distinguir os grupos normal, chagásico indeterminado e chagásico cardiopata entre si. Observou-se que a maioria dos índices (a)

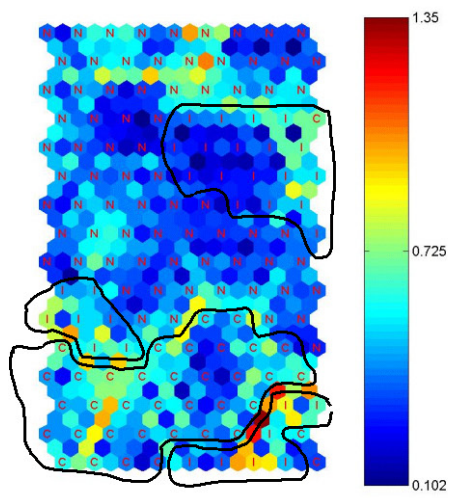

(c)

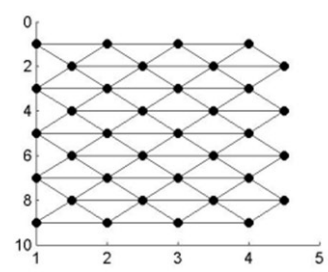

(e)

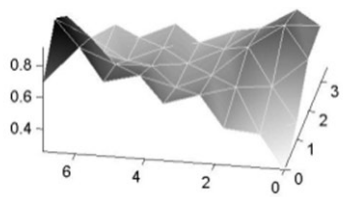

(g)

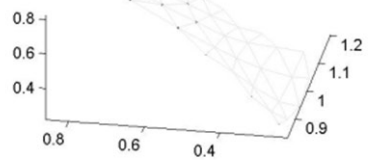

(i)

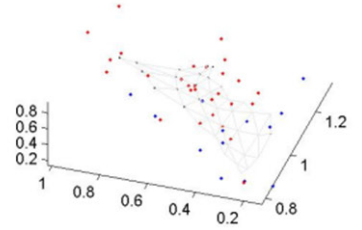

(b)

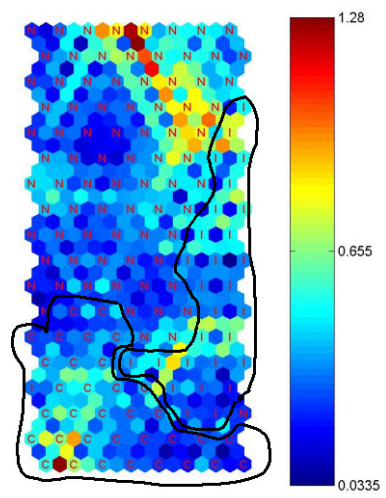

(d)

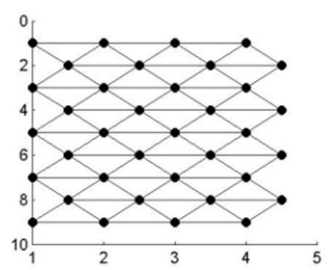

(f)

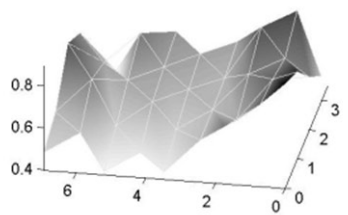

(h)

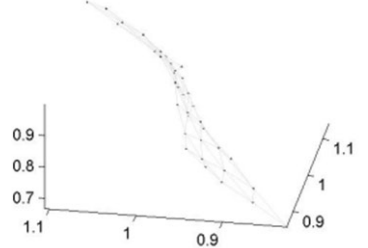

(j)

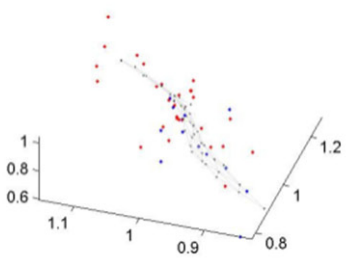

Figura 4. Configuração da rede neural tendo como entrada índices temporais (coluna da esquerda) ou índices escalográficos (coluna da direita): (a,b) mapa de Kohonen obtido após o treinamento com 50 épocas (N: normal; C: chagásico cardiopata; I: chagásico indeterminado); $(\mathrm{c}, \mathrm{d})$ grade do mapa; (e,f) matriz de distâncias; $(\mathrm{g}, \mathrm{h})$ vetores protótipos; e (i,j) dados obtidos após o treinamento com 50 épocas, superpostos aos vetores protótipos.

Figure 4. Neural network configuration using temporal indexes (left column) or scalographic indexes (rigt column): (a,b) Kohonen Map obtained after 50 training epochs ( $N$ : normal; $C$ : Chagas cardiomyopathy; I: indeterminate form of Chagas'disease); (c,d) map grid; (e,f) distance matrix; $(g, h)$ prototype vectors; and $(i, j)$ data after 50 training epochs, superimposed over the prototype vectors. 
Tabela 2. Avaliação dos índices temporais e escalográficos, com relação à significância da diferença estatística entre os três grupos de indivíduos estudados.

Table 2. Evaluation of the temporal and scalographic indexes, with respect to the statistical significance of the difference between the three groups studied in this work.

\begin{tabular}{lccc}
\hline & $\begin{array}{c}\text { Normais } \\
\text { vs. }\end{array}$ & $\begin{array}{c}\text { Normais } \\
\text { vs. }\end{array}$ & $\begin{array}{c}\text { Chagásicos indeterminados } \\
\text { vs. }\end{array}$ \\
Média & Shagásicos indeterminados & $\begin{array}{c}\text { Chagásicos cardiopatas } \\
\text { Chagásicos cardiopatas }\end{array}$ \\
Desvio padrão & $\mathrm{S}$ & $\mathrm{S}$ & $\mathrm{N}$ \\
Coeficiente de variação & $\mathrm{S}$ & $\mathrm{S}$ & $\mathrm{I}$ \\
pNN50 & $\mathrm{N}$ & $\mathrm{S}$ & $\mathrm{N}$ \\
RMSSD & $\mathrm{I}$ & $\mathrm{S}$ & $\mathrm{N}$ \\
Potência VLF média & $\mathrm{I}$ & $\mathrm{S}$ & $\mathrm{S}$ \\
Potência LF média & $\mathrm{N}$ & $\mathrm{S}$ & $\mathrm{N}$ \\
Potência HF média & $\mathrm{S}$ & $\mathrm{S}$ & $\mathrm{N}$ \\
Faixa dinâmica de potência VLF & $\mathrm{N}$ & $\mathrm{S}$ & $\mathrm{S}$ \\
Faixa dinâmica de potência LF & $\mathrm{N}$ & $\mathrm{N}$ & $\mathrm{N}$ \\
Faixa dinâmica de potência HF & $\mathrm{I}$ & $\mathrm{N}$ & $\mathrm{N}$ \\
Densidade do escalograma & $\mathrm{N}$ & $\mathrm{N}$ & $\mathrm{I}$ \\
\hline
\end{tabular}

S: $p<0,05 ;$ I: $0,05 \leq p<0,10 ; \mathrm{N}: p \geq 0,10$.

consegue discriminar o grupo normal do chagásico cardiopata, mas tem dificuldade para separar o normal do chagásico indeterminado e, principalmente, o chagásico indeterminado do chagásico cardiopata.

\section{Discussão}

Foi observado, por meio de distribuições tempoescala, que os pacientes chagásicos cardiopatas apresentam, efetivamente, uma redução da capacidade de modulação "fina" da função autonômica cardíaca (observada, como uma redução da energia na banda $\mathrm{HF}$, relacionada ao sistema parassimpático), conforme previsto na literatura.

Assim, foi verificado que o índice de potência média na banda HF do escalograma apresenta a capacidade de discriminar os grupos normal, chagásico indeterminado e chagásico cardiopata entre si. Observa-se que, de modo geral, os demais índices conseguem discriminar com facilidade o grupo normal do chagásico cardiopata, mas têm dificuldade para separar o normal do chagásico indeterminado e, principalmente, o chagásico indeterminado do chagásico cardiopata (tarefa esta realizada a contento pelo índice proposto).

Foi constatado, ainda, que as redes neurais escalográficas apresentam resultados de discriminação superiores às redes neurais temporais, o que comprova que, para o universo analisado, os índices escalográficos são melhores que os índices temporais para a classificação de pacientes em normais, chagásicos cardiopatas e chagásicos indeterminados, a partir de índices relacionados aos seus sinais de VFC. Esse resultado já era esperado, em face da capacidade de decorrelação de sinais e compactação de energia transformada wavelet DoG, fazendo com que a assinatura espectral de interesse seja enfatizada no domínio transformado.

Os resultados obtidos foram comparados com o trabalho similar mais recente (Escalera et al., 2008), onde foram obtidos resultados para a discriminação dos grupos de indivíduos saudáveis (grupo 0), pacientes com a Doença de Chagas sem sinais de cardiopatias na avaliação cardiográfica (grupo 1), pacientes com a Doença de Chagas com cardiopatias fracas ou moderadas, detectadas no ECG convencional (grupo 2) e pacientes com a Doença de Chagas com cardiopatias severas, detectadas no ECG convencional (grupo 3). Para os quatro classificadores propostos pelos autores, o melhor resultado foi obtido para o classificador Adaboost discreto com decodificação Loss-Weighted, que resultou em uma acurácia de classificação em torno de $72 \%$ para os três níveis de pacientes com a Doença de Chagas. Na Tabela 1, foram apresentados os resultados para a rede neural escalográfica, com erro de classificação inferior a 3\% para os grupos estudados. Entretanto, esses resultados não podem ser comparados diretamente, pois a inclusão do grupo 2 tornou o grau de dificuldade do experimento realizado por estes autores maior do que o do experimento aqui apresentado. Ademais, os resultados dos algoritmos com adaptação, como as redes neurais, para um conjunto de sinais similares de treinamento e teste, tendem a ser superiores aos dos algoritmos sem laço fechado, por conta do ajuste do algoritmo inteligente às características da base de 
sinais processada. Na continuação desta pesquisa, para avaliação da capacidade de classificação da rede neural escalográfica, propõe-se a realização de experimentos com bases de dados diferentes das bases utilizados no treinamento da rede neural.

Em conclusão, este trabalho apresentou e avaliou um sistema de apoio ao diagnóstico de pacientes chagásicos baseado em escalogramas e redes neurais auto-organizáveis. A metodologia proposta motrouse capaz de distinguir entre indivíduos normais, chagásicos cardiopatas e chagásicos indeterminados. Os índices escalográficos propostos mostraram maior capacidade classificatória que os índices temporais tradicionais. Em particular, o índice de potência média na banda HF do escalograma apresentou a maior capacidade discriminatória para os três grupos estudados.

A análise das distribuições tempo-escala de sinais de VFC mostrou que os pacientes chagásicos cardiopatas apresentam, efetivamente, uma redução da capacidade de modulação "fina" da função autonômica cardíaca (observada, como uma redução da energia na banda HF, relacionada ao sistema parassimpático), conforme previsto na literatura. Assim, propõe-se buscar índices escalográficos que indiquem o grau de perda de capacidade de modulação vagal nos pacientes chagásicos cardiopatas em comparação com os indivíduos normais.

\section{Referências}

Bifulco P, Cesarelli M, Bracale M. HRV adaptive spectral estimation for transient detection. In: Annual EMBS international Conference: Proceedings of the 22nd Annual EMBS international Conference; 2000 July 23-28, Chicago. Chicago: IEEE; 2000. p. 3165-7. http://dx.doi.org/10.1109/ IEMBS.2000.901559

Bootsma M, Swenne CA, Cats VM, Bruschke AVG. Pharmacological validation of heart rate variability measures that assess sympathetic or vagal tone or the sympathovagal balance. Computers in Cardiology. 1994; 1(1):301-4. http:// dx.doi.org/10.1109/CIC.1994.470189

Bravo R, Escalona O, Mora F, Passariello G. Late potential analysis in Chagas disease using the vector spectral combination of orthogonal leads. In: Annual International Conference of the IEEE: Proceedings of the 19th Annual International Conference of the IEEE; 1997. IEEE; 1997. p. 78-80. http://dx.doi.org/10.1109/IEMBS.1997.754468

Carranza R, Andina D. Wavelet-neural processing for computer aided diagnosis. In: Biannual World Automation Congress: Proceedings of the 5th Biannual World Automation Congress; 2002 June 9-13; Piscataway, EUA. IEEE; 2002. vol. 13, p. 215-20. http://dx.doi.org/10.1109/ WAC.2002.1049547

Cházaro A, Craven G, Eberhart R. Myocardial infarction diagnosis by a neural network. In: Conference of the
IEEE Engineering in Medicine and Biology Society: 20th Annual International Conference of the IEEE Engineering in Medicine and Biology Society; 1998 Oct 29; Hong Kong. IEEE; 1998. vol. 20, n. 3, p. 1121-4. http://dx.doi. org/10.1109/IEMBS.1998.747068

Correia D, Junqueira Jr LF, Molina RJ, Prata A. Cardiac autonomic modulationevaluated by heart interval variability is unaltered but subtly widespread in the indeterminate Chagas disease. Pacing and Clinical Electrophysiology. 2007; 30(6):772-80. PMid:17547611. http://dx.doi.org/10.1111/j.1540-8159.2007.00749.x

Correa R, Laciar P, Arini P, Jané R. Analysis of QRS Loop in the Vectorcardiogram of Patients with Chagas' Disease. Conference Proceedings - IEEE Engineering in Medicine and Biology Society. 2010; 2010:2561-4. http://dx.doi. org/10.1109/IEMBS.2010.5626863

Escalera S, Pujol O, Radeva P. Error-correcting output coding for chagasic patients characterization. In: ICPR 2008: Proceedings of the 19th International Conference on Pattern Recognition; 2008, Tampa. IEEE; 2008. p. 1-4. http://dx.doi. org/10.1109/ICPR.2008.4761243

Esteller R, Mora F, Bevilacqua G, Mendoza I, Marquez J, Lander P. Study of sudden death in Chagas disease: a proposed methodology. In: Engineering in Medicine and Biology Society, Engineering Advances: New Opportunities for Biomedical Engineers: Proceedings of the 16th Annual International Conference of the IEEE; 1994 Nov 03-6, Baltimore. IEEE; 1994. vol. 2, p. 916-7. http://dx.doi. org/10.1109/IEMBS.1994.415211

Guillén P, Vallverdd M, Jugo D, Carrasco H, Caminal P. Nonlinear dynamics in heart rate variability of normal subjects and chagasic Patients. In: Annual EMBS International Conference: Proceedings of the 22th Annual EMBS International Conference; 2000 July 23-28, Chicago. Chicago: IEEE; 2000. p. 2414-5. http://dx.doi.org/10.1109/IEMBS.2000.901285

Heidari H, Shahidi AV, Aminian K, Sadati N. Analysis of the sustained ventricular arrhythmias from SAECG using artificial neural network and fuzzy clustering algorithm. In: Conference of the IEEE Engineering in Medicine and Biology Society: 20th Annual International Conference of the IEEE Engineering in Medicine and Biology Society; 1998 Oct 29; Hong Kong. IEEE; 1998. vol. 20, n. 1, p. 104-7. http://dx.doi.org/10.1109/IEMBS.1998.745838

Junqueira Jr LF. Insights into the clinical and functional significance of cardiac autonomic dysfunction in Chagas disease. Revista da Sociedade Brasileira de Medicina Tropical. 2012; 45(2):243-52. http://dx.doi.org/10.1590/ S0037-86822012000200020

Kiviluoto K. Topology preservation in self-organizing maps. In: International Conference on Neural Networks: Proceedings of International Conference on Neural Networks; 1996, Washington. IEEE; 1996; p. 294-9. http://dx.doi.org/10.1109/ ICNN.1996.548907

Kleiger RE, Stein PK, Bigger JT Jr. Heart rate variability: measurement and clinical utility. Annals of Noninvasive Electrocardiology. 2005; 10(1):88-101. PMid:15649244. http://dx.doi.org/10.1111/j.1542-474X.2005.10101.x 
Kohonen T. Self-Organizing Maps. 2nd ed. Heidelberg: Springer; 1995. Series in Information Sciences, v. 30.

Lotric MB, Stefanovska A, Stajer D, Rovan V. Spectral components of heart rate variability determined by wavelet analysis. Physiology Measurements. 2000; 21(1):441-57. http://dx.doi.org/10.1088/0967-3334/21/4/302

Marmol-Herrera L, Warwick K. Reduction of invasive tests in chagasic patients with a modified self-organizing map. In: Annual EMBS International Conference: Proceedings of the 23rd Annual EMBS International Conference; 2001, Istanbul, Turkey. IEEE; 2001. p. 1676-9. http://dx.doi. org/10.1109/IEMBS.2001.1020537

Pueyo E, Laciar E, Anzuola E, Laguna P, Jané R. Assessment of myocardial damage in chronic chagasic patients using QRS slopes. Computers in Cardiology. 2007; 34:725-78. http://dx.doi.org/10.1109/CIC.2007.4745588

Reinhardt L, Vesanto R, Montonen J, Fetsch T, Makijarvi M, Sierra G, Breithardt G. Application of Learning Vector Quantization for localization of myocardial infarction. In: Annual International Conference of the IEEE Engineering in Medicine and Biology Society: Proceedings of 18th Annual International Conference of the IEEE Engineering in Medicine and Biology Society; 1996, Amsterdam, 1996. p. 921-2. http://dx.doi.org/10.1109/IEMBS.1996.652642

Rioul O, Flandrin P. Time-scale energy distributions: a general class extending wavelet transforms. IEEE Transactions on Signal Processing. 1992; 40(7):1746-57. http://dx.doi. org/10.1109/78.143446

Sapoznikov D, Luria MH, Gotsman MS. Heart rate variability in the low frequency range: periodic versus non-periodic fluctuations. Computers in Cardiology. 1994; 769-72. http:// dx.doi.org/10.1109/CIC.1994.470072

Souza Neto J. Análise de sinais de eletrocardiografia e de variabilidade da frequência cardíaca utilizando distribuições tempo-freqüência e tempo-escala [tese]. Brasília: Departamento de Engenharia Elétrica, Universidade de Brasília; 2003. 186 p.

Vasconcelos DF, Junqueira Jr LF. Distinctive impaired cardiac autonomic modulation of heart rate variability in chronic Chagas' indeterminate and heart disease. Journal of Electrocardiology. 2009; 42(3):281-9. PMid:19095247. http://dx.doi.org/10.1016/j.jelectrocard.2008.10.007

Vasconcelos DF, Junqueira Jr LF. Cardiac autonomic and ventricular mechanical functions in asymptomatic chronic chagasic cardiomyopathy. Arquivos Brasileiros de Cardiologia. 2011; 98(1):111-9.

Vizcardo M, Jiménez J, Moleiro F, Marcano A, Octavio A, Rodríguez A. Analysis of the Heart Rate Variability and Stratification of the Risk of Cardiac Patients with Chagas' Disease. Computers in Cardiology. 2007; 34:465-7. http:// dx.doi.org/10.1109/CIC.2007.4745523

Wiklund U, Akay M. Spectral analysis of bioelectric signals by adapted wavelet transform. In: Conference on Bioelectromagnetism: Proceedings of 2nd International Conference on Bioelectromagnetism; 1998, Melbourne. IEEE; 1998. p. 9-10. PMid:9536500. http://dx.doi. org/10.1109/ICBEM.1998.666369

Yang F, Liao W. Modeling and decomposition of HRV signals with wavelet transforms. IEEE Engineering in Medicine and Biology. 1997; 1(1):17-22. http://dx.doi. org/10.1109/51.603643

\footnotetext{
Autores

João Souza Neto, João Luiz Azevedo de Carvalho, Adson Ferreira da Rocha, Francisco Assis de Oliveira Nascimento* Grupo de Processamento Digital de Sinais - GPDS, Departamento de Engenharia Elétrica - ENE, Universidade de Brasília - UnB, Campus Universitário Darcy Ribeiro, Asa Norte, CP 4386, CEP 70910-900, Brasília, DF, Brasil

\section{João Souza Neto}

Universidade Católica de Brasília - UCB, SGAN 916, Módulo B, Av. W5, CEP 70790-160, Brasília, DF, Brasil

\section{Adson Ferreira da Rocha}

Faculdade UnB-Gama, Universidade de Brasília - UnB, Área Especial, Projeção A, Setor Leste Gama, CEP 72444-240, Brasília, DF, Brasil

\section{Luiz Fernando Junqueira Junior}

Laboratório Cardiovascular - LC, Faculdade de Medicina - FM, Universidade de Brasília - UnB, Campus Universitário Darcy Ribeiro, CEP 70910-900, Brasília, DF, Brasil
} 